\title{
Entropy effects during sorption of alkanes in zeolites
}

\author{
Rajamani Krishna, Berend Smit and Sofia Calero \\ Department of Chemical Engineering, University of Amsterdam, Nieuwe Achtergracht 166, 1018 \\ WV Amsterdam, The Netherlands.E-mail: krishna@science.uva.nl; Fax: + 3120 5255604; \\ Tel: 31205257007
}

Received 8th January 2002

First published as an Advance Article on the web 8th April 2002

Recent developments in Configurational-Bias Monte Carlo (CBMC) techniques allow the accurate calculation of the sorption isotherms for alkanes, and their mixtures, in various zeolites. The CBMC simulations give new insights into subtle entropy effects affecting mixture adsorption. Three types of entropy effects can be distinguished. (1) Size entropy effects favour the component with the smaller number of $\mathrm{C}$ atoms because the smaller molecule finds it easier to fill in the 'gaps' within the zeolite matrix at high molecular loadings. (2) Configurational entropy effects come into play for mixtures of alkanes that differ in the degree of branching. For a mixture of linear and branched alkanes with the same number of $\mathrm{C}$ atoms, configurational entropy effects favour the linear isomer because such molecules 'pack' more efficiently within, say, the intersecting channel topology of MFI zeolite. (3) Length entropy effects comes into force for sorption of linear and branched alkanes within the cylindrical channels of say AFI and MOR zeolites; here the double branched alkane has the shortest length and can be packed more efficiently within the channels. We demonstrate that CBMC simulations allow the efficient screening of zeolite structures for a given separation duty and aid the development of novel separation processes exploiting entropy effects.

\section{Introduction}

The separation of mixtures of alkanes is an important activity in the petroleum and petrochemical industries. For example, the

Rajamani Krishna (krishna@science.uva.nl) is Professor at the University of Amsterdam. He graduated in Chemical Engineering from the University of Bombay and was awarded a PhD degree in 1975 from the University of Manchester. He then joined the Royal Dutch Shell Laboratory in Amsterdam, where he was engaged in research, development and design of separation and reaction equipment. After nine years of industrial experience, Krishna returned to India to take over the Directorship of the Indian Institute of Petroleum. Since 1990 he has occupied the position of Professor of Chemical Reactor Engineering at the University of Amsterdam. His current research interests range from molecular modelling, bubble and particle dynamics and reactor scale up to process synthesis. Krishna has co-authored three texts, including Multicomponent Mass Transfer (Wiley, 1993). His research contributions have won him the Conrad Premie of the Royal Dutch Institution of Engineers in 1981, and the Akzo-Nobel prize in 1997.

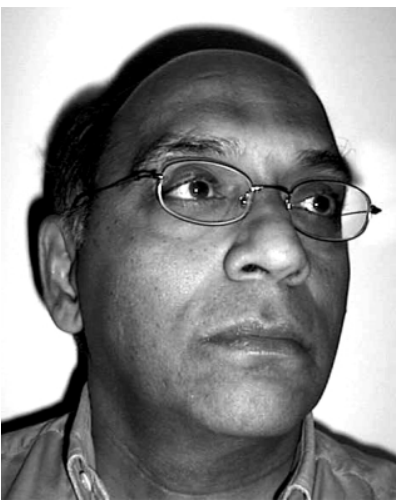

Rajamani Krishna

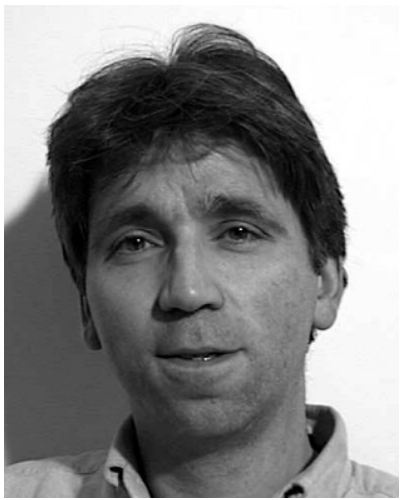

Berend Smit
Berend Smit (b.smit@science.uva.nl) is Professor at the University of Amsterdam. He graduated in Chemical Engineering from the Delft University of Technology in 1988 and subsequently joined the Royal Dutch Shell Laboratory in Amsterdam. His research work at Shell resulted in a PhD degree from the University of Utrecht in 1990. The focus of Smit's thesis was simulation of phase equilibria and surfactant self-assembly. In particular a theoretical foundation of the Gibbs-ensemble technique was developed. He then spent a year as Visiting Scientist at the University of California at Berkeley. Since 1997 Smit has been Professor of Computational Chemistry at Amsterdam. He is the co-author of Understanding Molecular Simulations (2nd edition, Academic Press, 2002). For his research contributions Smit received the Gold Medal of the Royal Dutch Chemical Society (KNCV) in 1997.

SofiaCalero (calero@science.uva.nl) is a postdoctoral researcher at the University of Amsterdam. She graduated in Chemistry from the University Complutense of Madrid in
1995. She was awarded a PhD

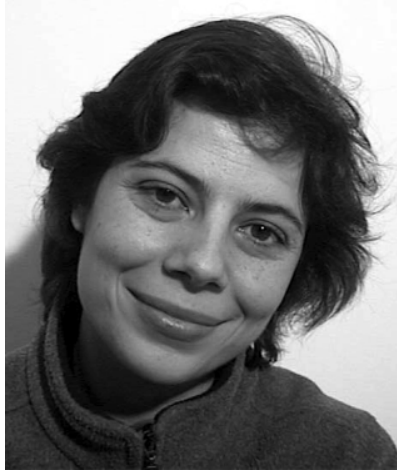

Sofia Calero for her thesis on the simulation of non-equilibrium properties of some linear polar fluids. From 1996 to 1999 she spent four trainee periods, respectively at the University of Sheffield (UK), University of Agricultural Sciences, (Vienna), University of Pennsylvania (Philadelphia) and ETHZ (Zurich). Currently, she is the holder of a Marie Curie Individual fellowship. 
products from a catalytic isomerization reactor consist of a mixture of linear, mono-methyl and di-methyl alkanes. Of these, the di-branched molecules are the most desired ingredients in petrol because they have the highest octane number. It is therefore required to separate the di-methyl alkanes and recycle the linear and mono-methyl alkanes back to the isomerization reactor. In the detergent industry, the linear alkanes are the desired components and need to be separated from the alkane mixture.

Selective sorption on zeolites is often used for separation of alkane mixtures. ${ }^{1-6}$ Zeolites are crystalline nanoporous materials that are used widely in the chemical industry as catalysts and adsorbents. A wide variety of zeolitic crystal structures are available, e.g. LTA, MFI, MEL, MOR, FAU, BEA, and these are used along with binders in the form of a pellet or coated on to a stainless steel support to form a zeolite membrane. The choice of the zeolite depends on the specific separation task in hand. For example, small-pore LTA zeolite is used for separation of linear alkanes using the molecular sieving principle; the branched molecules cannot enter the zeolite structure. Both linear and branched molecules are allowed inside the medium-pore MFI matrix and the sorption hierarchy in MFI will be dictated both by the alkane chain length and degree of branching. For the development of separation technologies we need to be able to calculate the mixture sorption characteristics for a wide range of operating conditions (pressures, temperatures, compositions). While there are several experimental studies on pure component isotherms, ${ }^{7-12}$ there is considerably less information on mixture isotherms. The lack of mixture sorption data is most probably due to the difficulty of experimentation. Mixture isotherms can be estimated from pure component isotherm data using the Ideal Adsorbed Solution Theory in some cases, but in general we need to account for thermodynamic non-ideality effects. ${ }^{3}$

Recent developments in Monte Carlo (MC) simulations have allowed the calculation of pure component and mixture sorption isotherms with only modest computational power. ${ }^{13-15}$ In MC simulations the zeolite crystals are allowed to exchange molecules with a reservoir of molecules at a fixed chemical potential in the Grand-Canonical (or $\mu, V, T$ ) ensemble; see Fig. 1. In this ensemble the temperature, volume and chemical

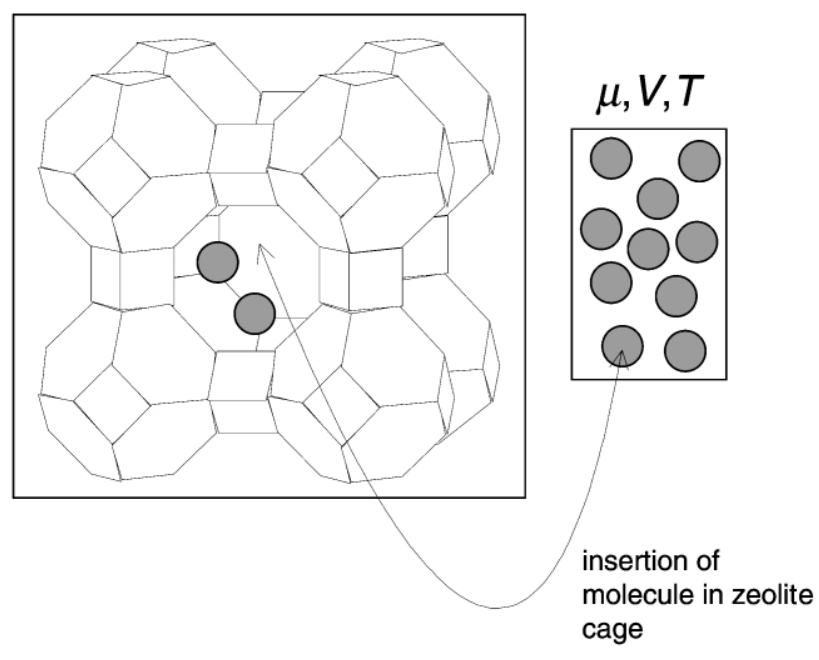

Fig. 1 Simulation of sorption of molecules in a zeolite using grandcanonical Monte Carlo techniques.

potential are fixed. The equilibrium conditions dictate that the temperature and chemical potential of fluid phase inside the zeolite and in the external reservoir must be equal. We therefore need to know only the $\mu$ and $T$ of the gas in the reservoir in order to determine the equilibrium concentration of molecules within the zeolite; this equilibrium is not affected by say the resistance of the gas-zeolite interface to transport of molecules. In a MC simulation one does not have to follow the 'natural' path of the molecule, as in a Molecular Dynamics (MD) simulation, and one can perform 'moves' to locate a molecule within an arbitrary position within the zeolite. This technique works very well for small molecules such as methane. In an MC simulation for methane sorption, we observe that out of 1000 attempts to place a methane molecule to a random position within a zeolite, 999 attempts will be rejected because the methane molecule overlaps with an atom within the zeolite matrix. For ethane, only 1 move in $10^{6}$ attempts will be successful. Clearly, the strategy of randomly inserting molecules within a zeolite matrix will not work for long chain alkanes. To make MC simulations of long chain molecules feasible, the Configurational-Bias Monte Carlo (CBMC) technique has been developed. ${ }^{15}$

We first review the principles of the CBMC technique. Later, we show that CBMC simulations can provide new insights into the sorption behaviours of alkanes in zeolites and provide clues to the development of novel separations relying on these insights.

\section{CBMC simulation technique}

The principle of the CBMC technique is to grow an alkane chain, atom by atom, instead of attempting to insert the entire molecule at random. Fig. 2 shows one of the steps in this

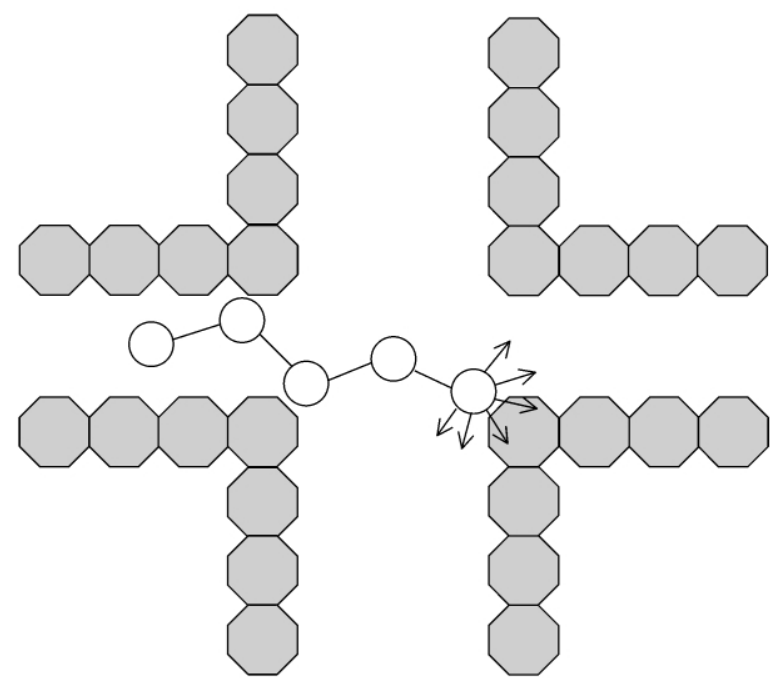

Fig. 2 Schematic drawing of the growing of an alkane in a zeolite in a CBMC move. The octagons represent the atoms of the zeolite and the circles represent the atoms of the alkane. Four atoms have been inserted successfully, and an attempt is made to insert the fifth.

algorithm; four atoms have been inserted successfully, and an attempt is made to insert the fifth. A number of candidate positions (denoted by arrows in Fig. 2) for insertion of the fifth atom are generated. Then one of the positions is selected in such a way that those trial positions with the lowest energy have the highest probability of being selected. Clearly a position which overlaps with the zeolite structure is not an acceptable candidate. Important to note is that this growing procedure introduces a bias, such that only the most favorable configurations are being generated. If one were to use the ordinary Metropolis acceptance rule, such a bias in the configurations of the molecules would lead to an incorrect distribution of configurations. This bias can be removed exactly by adjusting the acceptance rules. ${ }^{15}$ Computational details of the implementation of the CBMC algorithm are to be found in Vlugt et al. ${ }^{16,17}$

These simulations are performed in cycles and in each cycle, an attempt to perform one of the following moves is made: (1) displacement of a randomly selected chain; (2) rotation of a chain around its center of mass; (3) partial regrowth of a chain- 
a chain is selected at random and part of the molecule is regrown using the CBMC scheme; (4) exchange with a reservoir that fixes the chemical potential of the components-it is decided at random whether to add or to remove a molecule from the zeolite following the acceptance rules derived in Vlugt et al.;16,17 (5) change of identity (only in the case of mixtures) - one of the components is selected at random and an attempt is made to change its identity. ${ }^{16,17}$ This type of move has as imporant practical advantage that in step (4) one only has to exchange the smallest molecules with the reservoir.

Typically the number of MC cycles performed is $10^{7}$. Of these, $15 \%$ are displacements, $15 \%$ rotations, $15 \%$ partial regrowths, $50 \%$ exchanges with the reservoir and the remaining $5 \%$ of the moves are attempts to change the identity of a molecule. These probabilities can be further optimized depending on the details of the simulations.
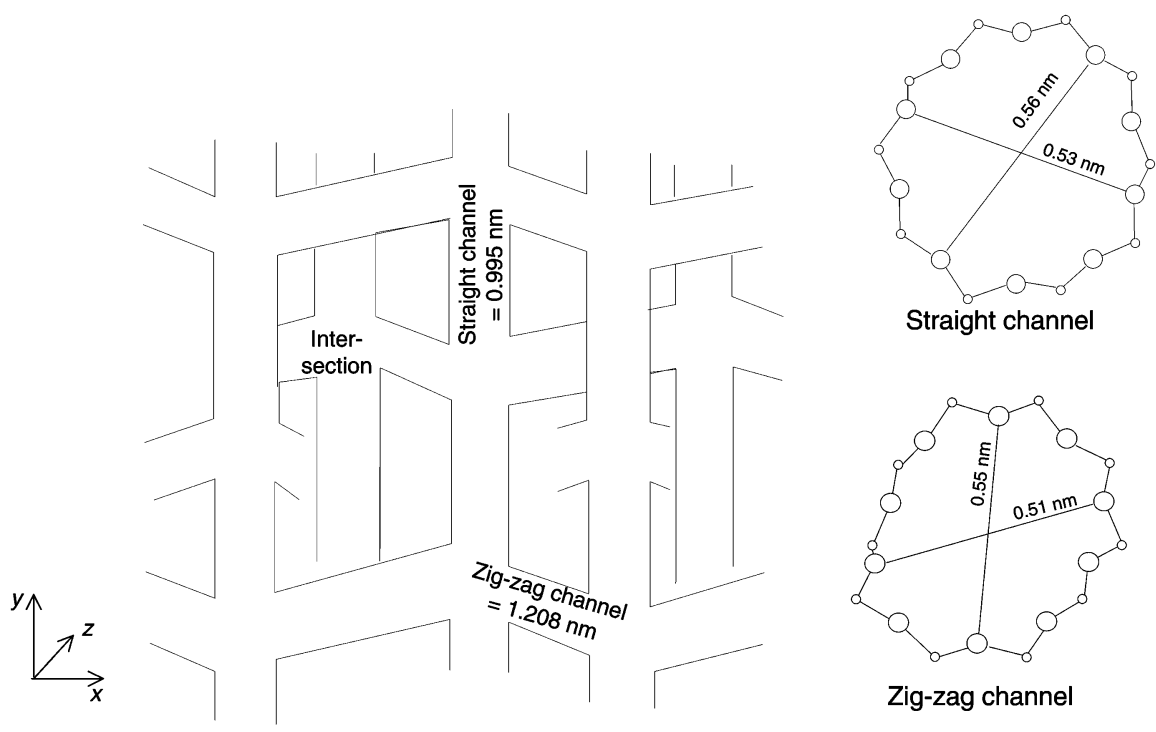

Fig. 3 Schematic of structure of MFI (silicalite-1). The cross-sections of the straight and zig-zag channels are shown in the inset.

(a) methane

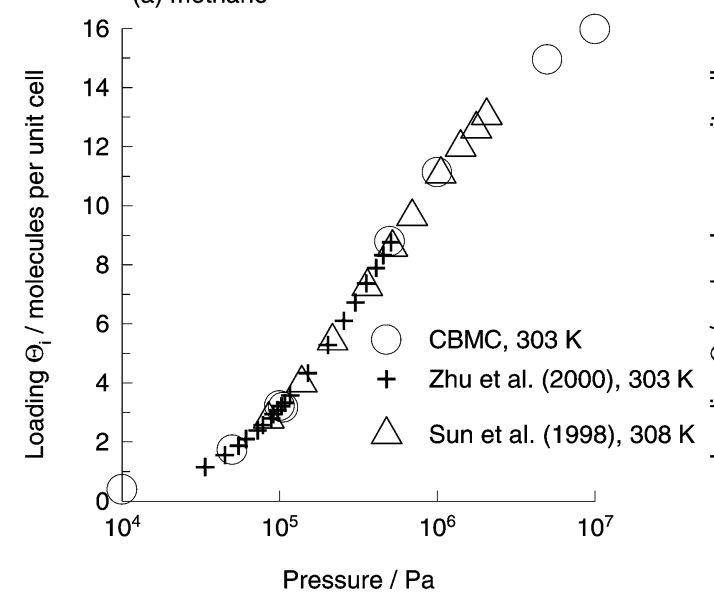

(c) propane

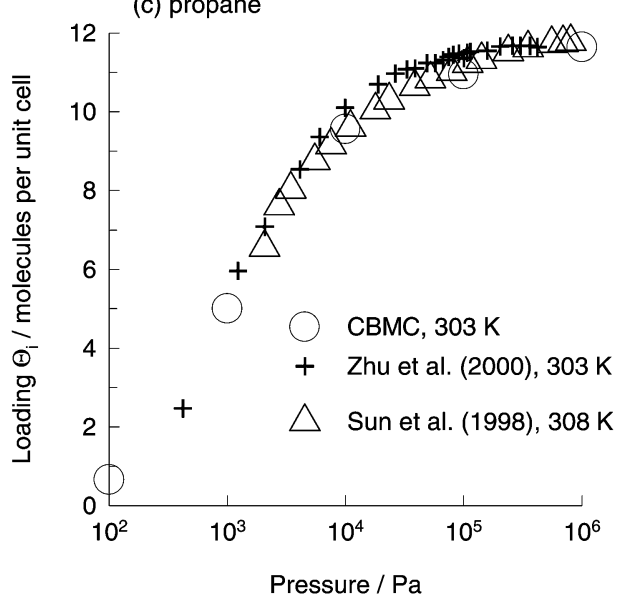

(b) ethane

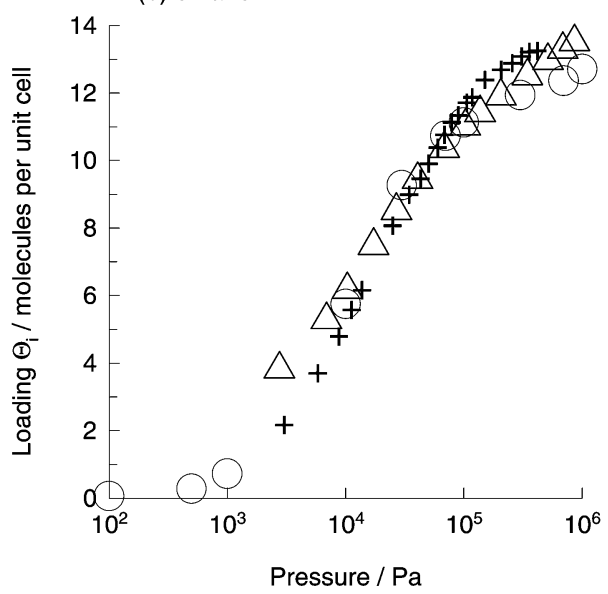

(d) n-butane

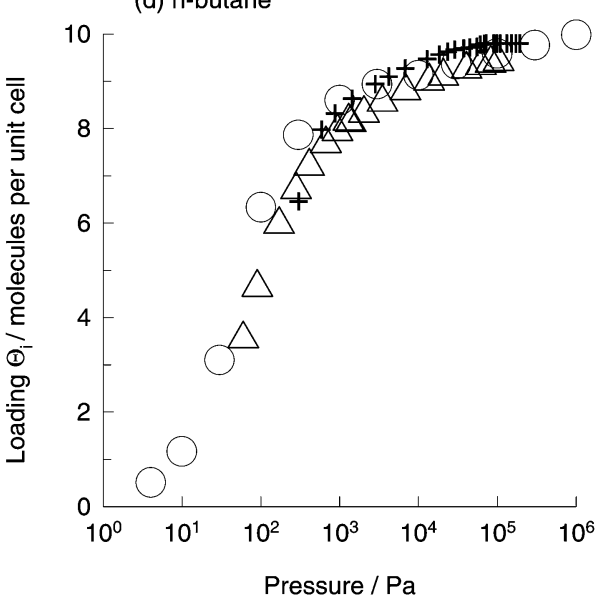

Fig. 4 Comparison of the experimental data ${ }^{25,26}$ for pure component isotherms for (a) methane, (b) ethane, (c) propane, and (d) n-butane in MFI at $300 \mathrm{~K}$ with CBMC simulations. ${ }^{22-24}$ 
(a) CBMC simulations vs Experiments

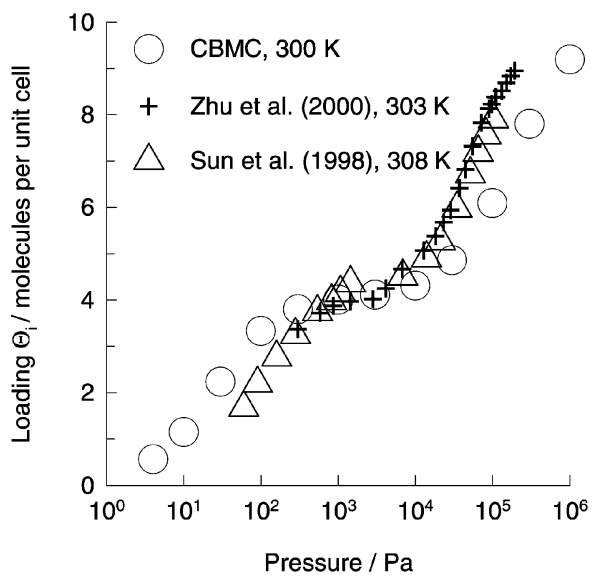

(b) isobutane siting

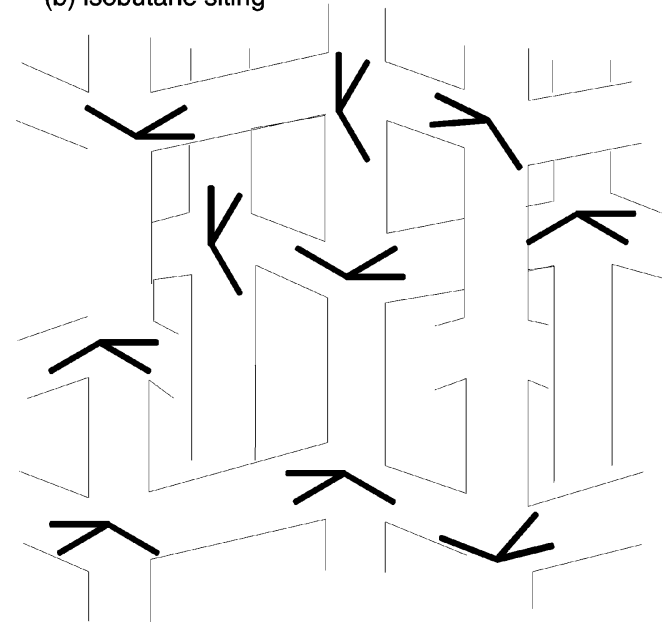

Fig. 5 (a) Comparison of the experimental data ${ }^{25,26}$ for pure component isotherm for isobutane in MFI at $300 \mathrm{~K}$ with CBMC simulations. ${ }^{22-24}$ (b) Schematic showing preferential location of isobutane molecules at the intersections between the straight and zig-zag channels.

A simple approach to describe the alkane molecules is to use the united-atom model, in which $\mathrm{CH}_{3}, \mathrm{CH}_{2}$, and $\mathrm{CH}$ groups are considered as single interaction centers. When these pseudoatoms belong to different molecules or to the same molecule but separated by more than three bonds, the interaction is given by a Lennard-Jones potential. The Lennard-Jones parameters are chosen to reproduce the vapor-liquid curve of the phase diagram as shown in Siepmann et al. ${ }^{18}$ The bonded interactions include bond-bending and torsion potentials; details for the alkane model can be found in Vlugt et al. ${ }^{16,17}$

For the calculation of the sorption isotherms of alkanes in silicalite, Macedonia and Maginn ${ }^{19}$ have adopted a more detailed all-atom model to represent the alkanes. Both the united-atom model and the all-atom model gave comparable results for the prediction of sorption of $\mathrm{C} 1-\mathrm{C} 3$ alkanes in silicalite. ${ }^{19}$

Most simulation studies follow the approach of Kiselev and co-workers ${ }^{20}$ and assume the zeolite lattice to be rigid and that interactions of an alkane with the zeolite are dominated by the dispersive forces between pseudo atoms of the alkane (within the framework of the united-atom description) and the oxygen atoms of the zeolite. In the Kiselev model the total interaction energy is represented by two terms, an attractive term and a repulsive term, and is described by a Lennard Jones potential; 16,17 the potential parameters are usually obtained by fitting to experimental heats of adsorption and Henry coefficients. In the more recent $\mathrm{PN}$ intermolecular potential model of Nicholson and co-workers, ${ }^{21}$ effects of polarizability and fixed charges in the zeolite matrix can be accounted for, but at significantly higher computational cost.

\section{Entropy effects during sorption}

\subsection{Sorption of pure alkanes}

Consider CBMC simulations for sorption of alkanes in the 1-4 $\mathrm{C}$ atom range in MFI zeolite, specifically silicalite- $1.22-24 \mathrm{Fig} .3$ shows a schematic of the structure of MFI that consists of a system of intersecting channels composed of zig-zag channels along $x$, cross-linked by straight channels along $y$. Both channels are defined by 10-rings. The straight channels are approximately elliptical in shape having a $0.53 \mathrm{~nm} \times 0.56 \mathrm{~nm}$ cross section while the zig-zag channels have a $0.51 \mathrm{~nm} \times 0.55$ $\mathrm{nm}$ cross section. The pure component isotherms at $300 \mathrm{~K}$ for methane, ethane, propane and n-butane are shown in Fig. 4. The CBMC simulations are seen to be in very good agreement with the experimental data of Sun et al. ${ }^{25}$ and Zhu et al. ${ }^{26}$ Such good agreement of CBMC simulations with measured isotherm data for pure alkanes is typical. ${ }^{17,27}$

CBMC simulations provide much more insight into sorption behaviour than is available from experiments alone. In order to illustrate this let us consider the experimental data for sorption of isobutane in MFI at 300-303 K; ${ }^{25,26}$ the sorption isotherm shows a pronounced inflection at a loading of 4 molecules per unit cell [see Fig. 5(a)]. This inflection behaviour is nicely reproduced by CBMC simulations. Up to a system pressure of $1 \mathrm{kPa}$, the isobutene molecules are exclusively located at the intersections, that have a maximum capacity of 4 molecules per unit cell [see the schematic in Fig. 5(b)]. This means that zeolite loadings in excess of 4 molecules per unit cell can only be achieved by pushing isobutene into the straight and zig-zag channels. Only when the pressure is significantly increased beyond $10 \mathrm{kPa}$ do the zig-zag channels and straight channels tend to get occupied. Due to its branched configuration, isobutane demands an extra 'push' to locate within these channels. This extra push is the root cause of the inflection behaviour.

Interestingly, n-hexane also shows a slight inflection at a loading of 4 molecules per unit (see e.g. Fig. 6); this inflection

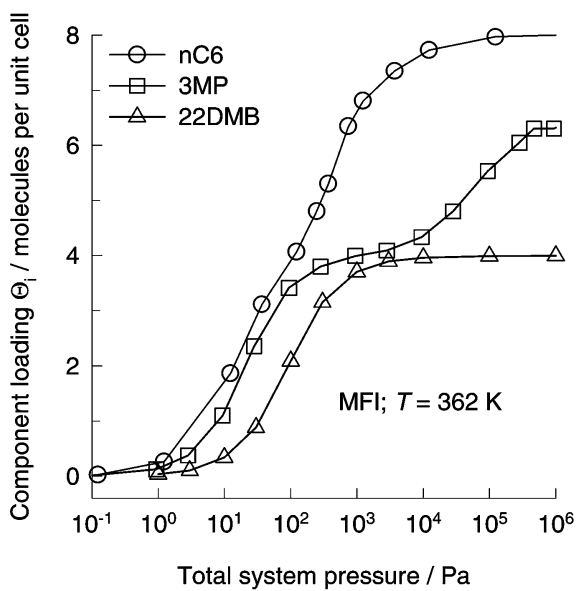

Fig. 6 (a) CBMC simulations of pure component isotherms for n-hexane, 3-methylpentane and 2,2-dimethylbutane in MFI at $362 \mathrm{~K}$. Data from refs. 27-30.

is due to 'commensurate' freezing ${ }^{31}$ caused by the fact that the length of the n-hexane molecule is commensurate with the length of the zig-zag channel [see Fig. 7(a)]. All mono-branched alkanes in the 5-8 C atom range are found to exhibit inflection behaviour, ${ }^{17,27-30}$ witness for example the inflection behaviour 
(a) nC6 siting

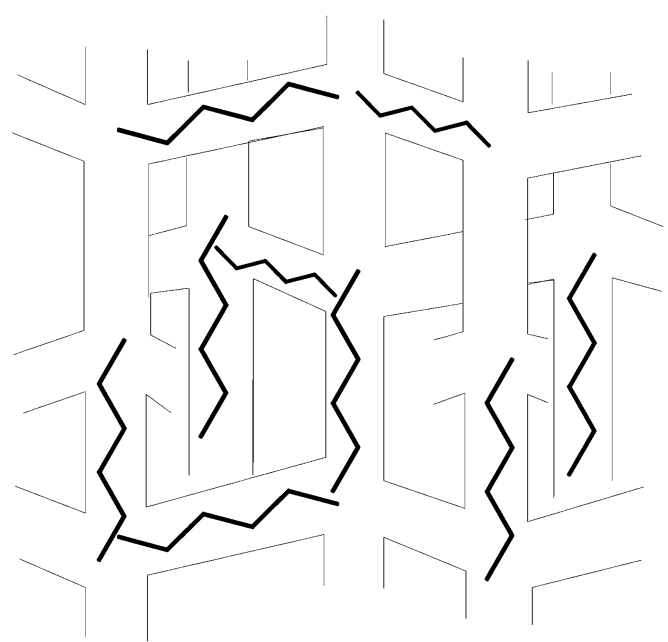

(b) 3MP siting

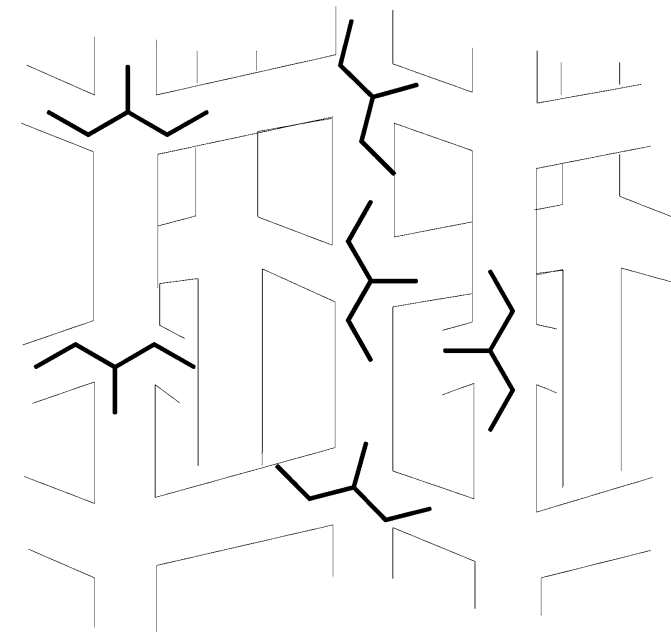

Fig. 7 (a) Schematic showing siting of n-hexane molecules within the MFI network. (b) Schematic showing preferential location of 3-methylpentane (3MP) at intersections between straight and zig-zag channels of MFI network.

exhibited by 3-methylpentane (3MP) in MFI zeolite [see Fig. 6]. Just as in the case of isobutane, 3MP prefers to locate at the intersections [see Fig. 7(b)] due to the availability of extra 'leg room'. Di-branched alkanes, typified by 2,2-dimethyl butane (22DMB), also prefer to locate at the intersections of MFI. However, these molecules are much bulker than monobranched alkanes and, consequently, they cannot be pushed into the channel interiors. The maximum loading of di-branched alkanes such as 22DMB, in MFI is restricted to 4 molecules per unit cell [see Fig. 6].

The inflection behaviour of mono-branched alkanes in MFI at a loading of 4 molecules per unit cell, as well as the restriction of the maximum loading of di-branched alkanes to this loading, is a consequence of configurational differences. This configurational entropy effect causes the saturation loadings of hexane isomers in MFI to follow the hierarchy linear > mono-branched $>$ di-branched.

With other zeolite structures the configurational entropy effects may act in a completely different manner. Consider the sorption of hexane isomers nC6, 3MP and 22DMB in AFI. The CBMC simulations of the sorption isotherms at $403 \mathrm{~K}$ are shown in Fig. 8. The sorption hierarchy, at saturation loading, is

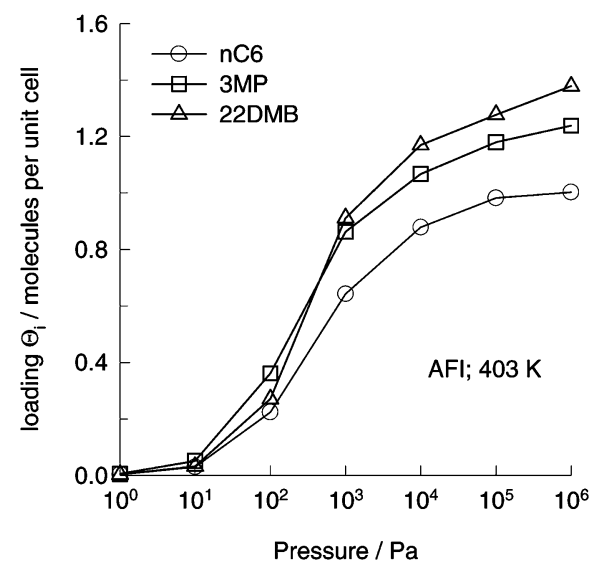

Fig. 8 Sorption isotherms for nC6, 3MP and 22DMB in AFI at $403 \mathrm{~K}$ determined by CBMC simulations.

found to be di-branched $>$ mono-branched $>$ linear which is opposite to the hierarchy for MFI. AFI consists of cylindrical channels of $0.73 \mathrm{~nm}$ diameter. The channel dimension is large enough to accommodate the bulky 22DMB and there is therefore no configurational penalty for these molecules.
However, the length of the molecules decreases with increased degree of branching (see Fig. 9); this implies that the number of

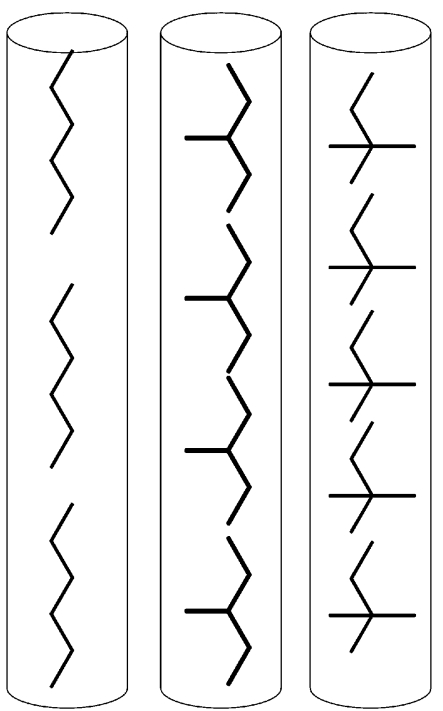

Fig. 9 Schematic of length entropy effect during sorption of nC6, 3MP and 22DMB in the cylindrical channels of AFI.

molecules that can be accommodated into the channels increases with the degree of branching. The increased sorption strength with increased branching can be termed a length entropy effect, arising as it does with decreasing linear dimension of the molecule. The length entropy effect has also been highlighted by Talbot ${ }^{32}$ in a more general, but idealized, manner.

\subsection{Size entropy effects during mixture sorption}

Almost all applications of adsorption involve mixtures, yet the number of experimental studies on adsorption of mixtures is very limited due to the difficulty of experimentation. Often in practice, the sorption selectivity of mixtures is estimated on the basis of the values of the Henry coefficients, i.e. the sorption strengths at near-zero loadings. ${ }^{33,34} \mathrm{We}$ shall demonstrate below that using Henry coefficients to estimate the sorption selectivity of mixtures can lead to completely wrong conclusions regarding separation possibilities.

Let us first consider sorption of a 95:5 mixture of methane (C1) and n-butane (nC4) in MFI at $300 \mathrm{~K}$. The component 
(a) 95-5 mixture isotherm

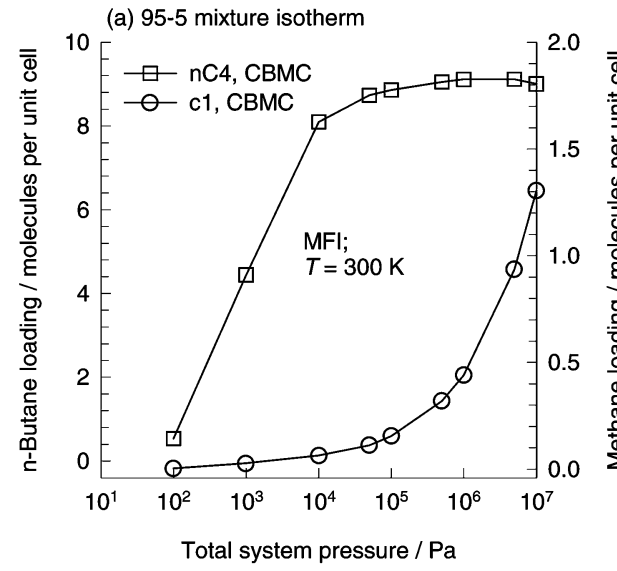

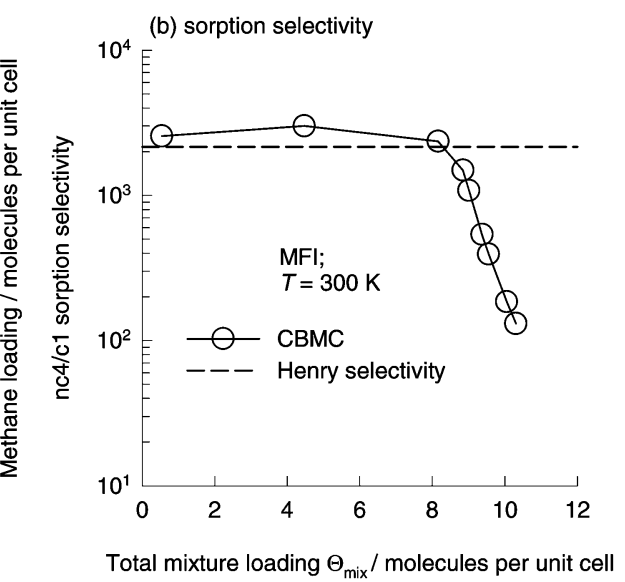

Fig. 10 (a) Sorption loadings of $95: 5$ of $\mathrm{C} 1$ and $\mathrm{nC} 4$ in MFI at $300 \mathrm{~K}$. (b) nC4/C1 sorption selectivity. CBMC simulation results of Krishna and Paschek. ${ }^{24}$

loadings in the mixture, obtained from CBMC simulations, ${ }^{24}$ are shown in Fig. 10(a). The sorption selectivity, defined by $S=$ $\left(\Theta_{1} / \Theta_{2}\right) /\left(p_{1} / p_{2}\right)$ where $\Theta_{\mathrm{i}}$ and $p_{\mathrm{i}}$ represent, respectively, the component loading and partial pressure in the gas phase, is shown in Fig. 10(b) as a function of the mixture loading. It is interesting to note that the sorption selectivity for $\mathrm{nC} 4$ with respect to $\mathrm{C} 1$ decreases significantly from the value dictated by the Henry coefficients (indicated by dashed line) when the mixture loading $\Theta_{\text {mix }}$ is increased beyond 8 molecules per unit cell. The reason for this selectivity decrease lies in the fact that at near saturation loadings the vacant sites within MFI are more easily filled up with the smaller $\mathrm{C} 1$ molecule than with the bulkier nC4 molecules. This is a size entropy effect.

MFI membrane permeation data of $\mathrm{C} 1-\mathrm{C} 2, \mathrm{C} 1-\mathrm{C} 3, \mathrm{C} 2-\mathrm{C} 4$, and $\mathrm{C} 1-\mathrm{nC} 4$ mixtures in published literature ${ }^{35-37}$ provides confirmation of the importance of size entropy effects at high loadings. In Fig. 11 we compare the sorption selectivity from

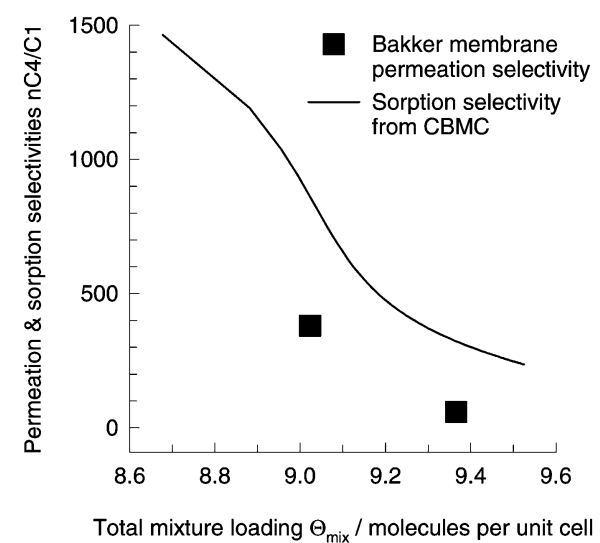

Fig. 11 Comparison of $\mathrm{nC} 4 / \mathrm{C} 1$ sorption selectivity in MFI at $300 \mathrm{~K}$ from CBMC simulations $^{24}$ with permeation selectivity from experimental data of Bakker. ${ }^{37}$

CBMC simulations with the permeation selectivity from the MFI permeation selectivity data of Bakker. ${ }^{37}$ We note that the strong decrease in the $\mathrm{nC} 4 / \mathrm{C} 1$ sorption selectivity with increased mixture loading is reflected in a corresponding decrease in the permeation selectivity. Clearly size entropy effects cannot be ignored in practice.

\subsection{Configurational entropy effects in mixture sorption}

Let us now consider the separation of hexane isomers, an important separation problem in the petroleum industry. ${ }^{4-6}$ Based on the sorption selectivity in MFI dictated by the experimentally determined Henry coefficients reported by Denayer, ${ }^{33}$ we would conclude that it is not possible to separate the linear and the mono-branched alkanes with high selectivities. In order to test this conclusion let us consider CBMC simulations $^{14,27}$ for sorption of a 50:50 mixture of $n-C_{6}$ and $3 \mathrm{MP}$ in MFI at a temperature of $362 \mathrm{~K}$. The component loadings in the mixture are shown in Fig. 12(a) for a range of pressures. It is interesting to note the maximum in the loading of $3 \mathrm{MP}$ at about $100 \mathrm{~Pa}$. When the pressure is raised above $100 \mathrm{~Pa}$ the loading of $3 \mathrm{MP}$ reduces virtually to zero. The $\mathrm{nC}_{6}$ molecules fit nicely into both straight and zig-zag channels [see Fig. 7(a)] whereas the 3MP molecules are preferentially located at the intersections between the straight channels and the zig-zag channels [Fig. 7(b)]. Below a total loading of 4 molecules per unit cell, there is no real competition between nC6 and 3MP. The nC6 locates within the channels and 3MP at the intersections. When all the intersection sites are occupied, to further adsorb 3MP we need to provide an extra 'push'. Energetically, it is more efficient to obtain higher mixture loadings by 'replacing' the $3 \mathrm{MP}$ with $\mathrm{nC}_{6}$; this configurational entropy effect is the reason behind the curious maxima in the $3 \mathrm{MP}$ loading in the mixture. The nC6/3MP sorption selectivity is plotted in Fig. 12(b). We see that the sorption selectivity increases from near-unity values for pressures below $100 \mathrm{~Pa}$ to values of around 50 near saturation loadings. Clearly, the Henry selectivity values [denoted by the dashed lines in Fig. 12(b)] are too pessimistic and ignore a potentially attractive separation possibility at high mixture loadings.

For an equimolar ternary mixture of nC6, 3MP and 22DMB configurational entropy effects act in such a way as to exclude both branched isomers from the MFI zeolite as the pressure is increased; see the CBMC simulations at $362 \mathrm{~K}$ shown in Fig. 13(a). The loadings of the three components follow 'normal' behaviour for pressures below $50 \mathrm{~Pa}$, i.e. follow the hierarchy dictated by the Henry coefficients. At a pressure of $500 \mathrm{~Pa}$, the total mixture loading is 4 molecules per unit cell; at this loading all the intersection sites are fully occupied. At pressures exceeding $500 \mathrm{~Pa}$, the MFI matrix prefers to accept $\mathrm{nC}_{6}$ into its matrix rather than either of the branched isomers. The loadings of both 3MP and 22DMB decrease as the pressure is increased beyond $500 \mathrm{~Pa}$. There are three types of entropic 'battles' in play at pressures exceeding $500 \mathrm{~Pa}$. The battle between linear isomer and its branched isomers is won by the linear isomer. The battle between the mono-methyl and di-methyl isomer is won by the mono-methyl isomer. The $n-\mathrm{C}_{6} / 3 \mathrm{MP}$ and $3 \mathrm{MP} /$ 22DMB sorption selectivities both increase significantly, beyond the Henry selectivity values (indicated by the dashed lines), when the total mixture loading exceeds 4; see Fig. 13(b). Direct experimental verification of the validity of these simulations is provided by the experimental data of Santilli. 38,39 

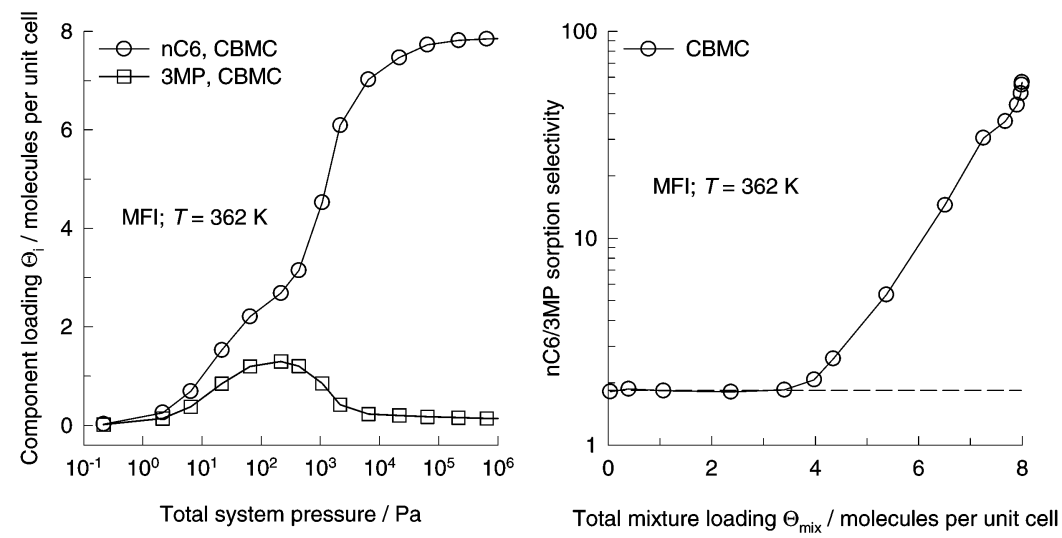

Fig. 12 (a) CBMC simulations of 50:50 mixture isotherm for nC6-3MP in MFI at 362 K. (b) Sorption selectivity as a function of total system pressure. The sorption selectivity based on Henry coefficients is indicated by the dashed line.

(a) Component loadings vs total pressure

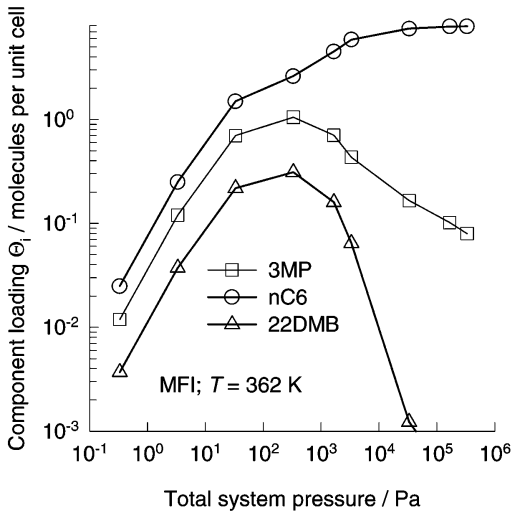

(b) Sorption selectivity vs total loading

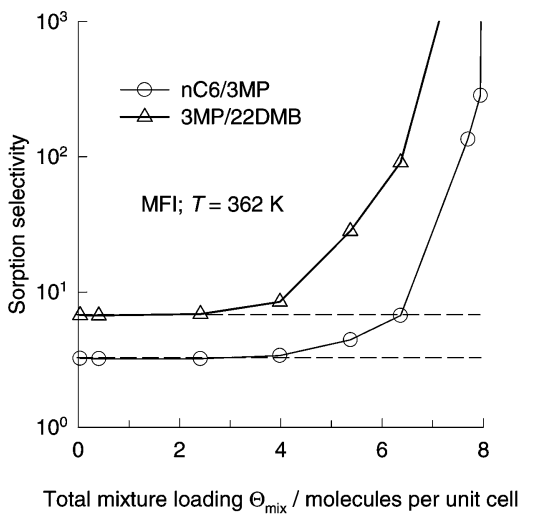

Fig. 13 (a) CBMC simulations ${ }^{39}$ of loadings for equimolar mixture of $\mathrm{nC}_{6}-3 \mathrm{MP}-22 \mathrm{DMB}$ in MFI at $362 \mathrm{~K}$. (b) Sorption selectivities as function of total loading.

The sorption hierarchy in MFI for a 5-component equimolar mixture of hexane isomers consisting of nC6, 2-methylpentane (2MP), 3MP, 22DMB and 2,3-dimethylbutane (23DMB) at high mixture loading is $\mathrm{nC} 6>>2 \mathrm{MP} \approx 3 \mathrm{MP}>>22 \mathrm{DMB} \approx$ 23DMB; see CBMC calculation results in Fig. 14(a). Hexane isomers can therefore be separated into three fractions linear, mono-branched and di-branched alkanes. This is exactly the hierarchy in which these components are eluted in a pulsed chromatographic test; ${ }^{40}$ see Fig. 14(b). But the important objective from a practical point of view to achieve such a separation hierarchy starting with a mixture say in the 5-7 C atoms range. $\mathrm{CBMC}$ simulations for mixture of linear, monobranched and di-branched alkanes in the 5-7 $\mathrm{C}$ atom range in MFI at $300 \mathrm{~K}$ are shown in Fig. 15. At low loadings (i.e. low pressures) we see that the sorption hierarchy is dictated by the number of $\mathrm{C}$ atoms, i.e. $\mathrm{C} 7$ isomers $>>\mathrm{C} 6$ isomers $>>\mathrm{C} 5$ isomers. Operating at low loadings does not yield the desired separation depending on the degree of branching. However, for mixture loadings approaching 8 molecules per unit cell, the situation changes dramatically; see the right end of Fig. 15. We see that the separation hierarchy is now linear $>>$ monobranched $>>$ di-branched, exactly as desired. This separation potential is of immense importance in the petroleum industry. ${ }^{4-6}$

Molecular simulations can also help us to screen promising zeolite structures for a given separation task, relying on differences in component loadings at equilibrium. For the separation of a 50:50 mixture of nC6 and 2MP, the nC6/2MP sorption selectivity in six different zeolite topologies, MFI, MEL, FAU, BEA, MOR and AFI are shown in Fig. 16. In FAU, which has large cages, entropy effects do not come into play and the separation selectivity is about unity over the whole pressure range. Large pore BEA also cannot be used to separate the hexane isomers. For the intersecting channel structures of medium pore size structures MFI and MEL, configurational entropy effects favour the linear isomer at mixture loadings exceeding 4 molecules per unit cell. In AFI and MOR, consisting of straight cylindrical channels, length entropy effects favour 2MP at high mixture loadings. To emphasise this further, in Fig. 17 we present CBMC simulations of a 5-component mixture of nC6, 2MP, 3MP, 22DMB and 23DMB in AFI and MOR at $300 \mathrm{~K}$. In AFI the sorption hierarchy at high mixture loadings is $23 \mathrm{DMB}>22 \mathrm{DMB} \approx 3 \mathrm{MP}>2 \mathrm{MP}>>$ nC6; this hierarchy is opposite of that observed in Fig. 14 for MFI. In MOR, the sorption hierarchy at high mixture loadings is $23 \mathrm{DMB}>22 \mathrm{DMB} \approx 3 \mathrm{MP} \approx 2 \mathrm{MP}>>$ nC6. The sorption hierarchy in AFI and MOR are dictated by length entropy effects.

\section{Concluding remarks}

In this review we have demonstrated the power of CBMC simulations in determining the adsorption isotherms for pure alkanes and their mixtures in various zeolites. Besides providing useful data for zeolite process design, molecular simulations provide insights not possible by experiments alone. The inflection in the isotherm of branched alkanes in MFI, is due to preferential location at the intersections between straight and zig-zag channels. CBMC simulations also help to highlight subtle entropy effects. For binary mixtures of linear alkanes, size entropy effects come into play at high mixture loadings and these counter chain length effects to reduce separation selectiv- 
(a) CBMC simulations of 5-component loadings

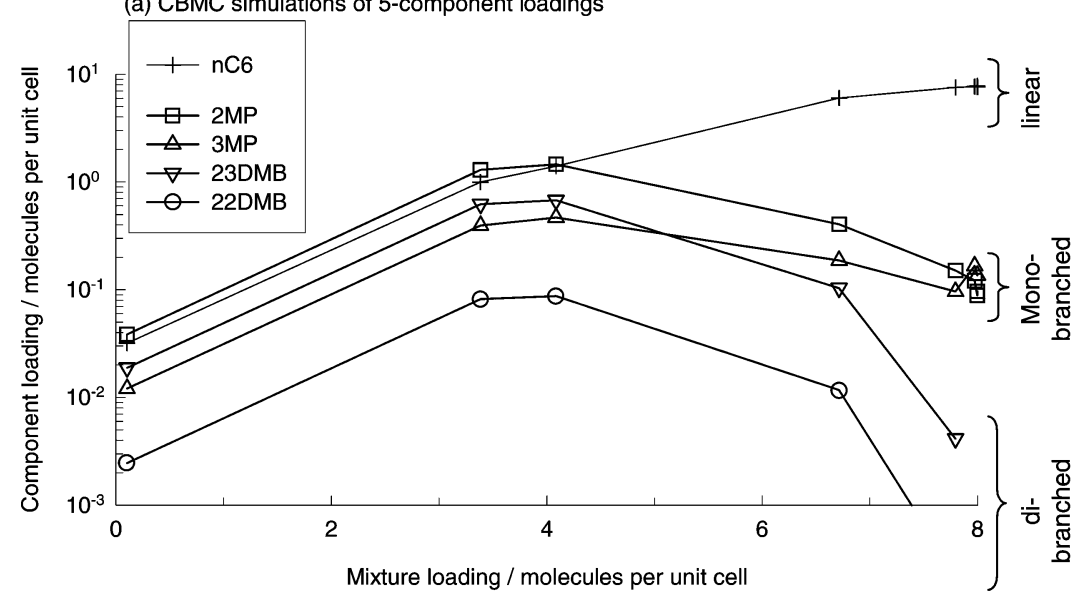

(b) Elution of hexane isomers in pulsed chromatographic column

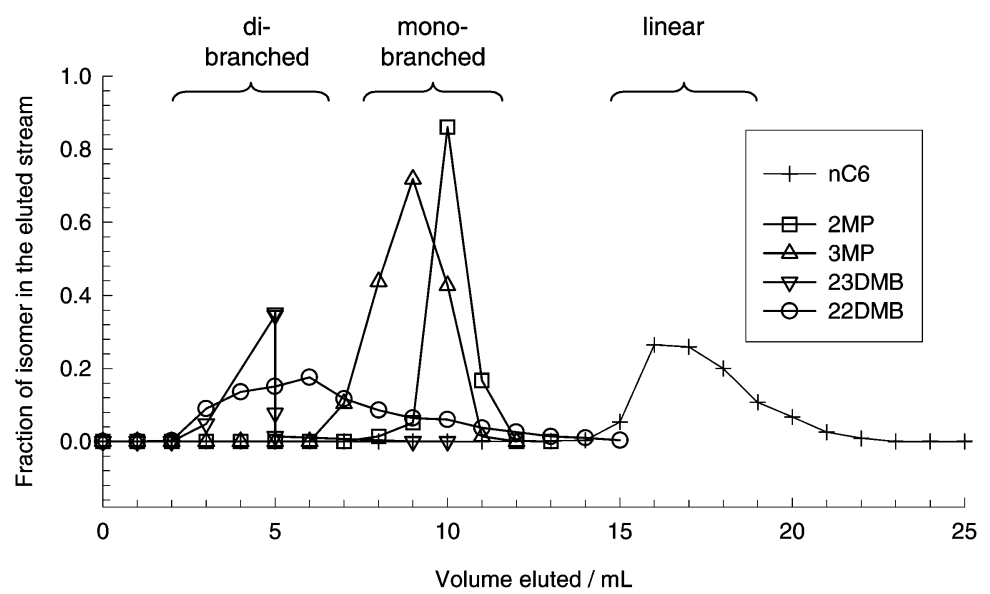

Fig. 14 (a) CBMC simulations of component loadings for equimolar mixture of $\mathrm{nC}_{6}, 2 \mathrm{MP}, 3 \mathrm{MP}, 22 \mathrm{DMB}$ and $23 \mathrm{DMB}$ in $\mathrm{MFI}$ at $300 \mathrm{~K}$. (b) Elution profiles in a pulsed chromatographic experiment .40

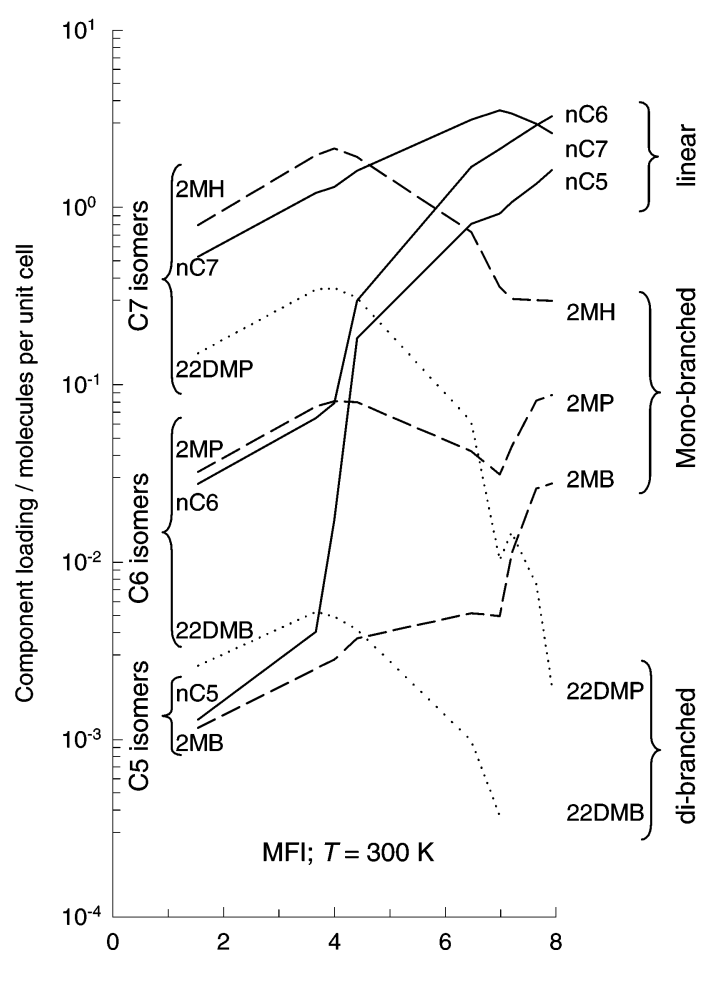

Total mixture loading $\Theta_{\text {mix }} /$ molecules per unit cell

Fig. 15 Component loadings in an equimolar 8-component mixture nC52MB-nC6-2MP-22DMB-nC7-2MH-22DMP at $300 \mathrm{~K}$ in MFI as a function of total mixture loading $\Theta_{\text {mix }}$.

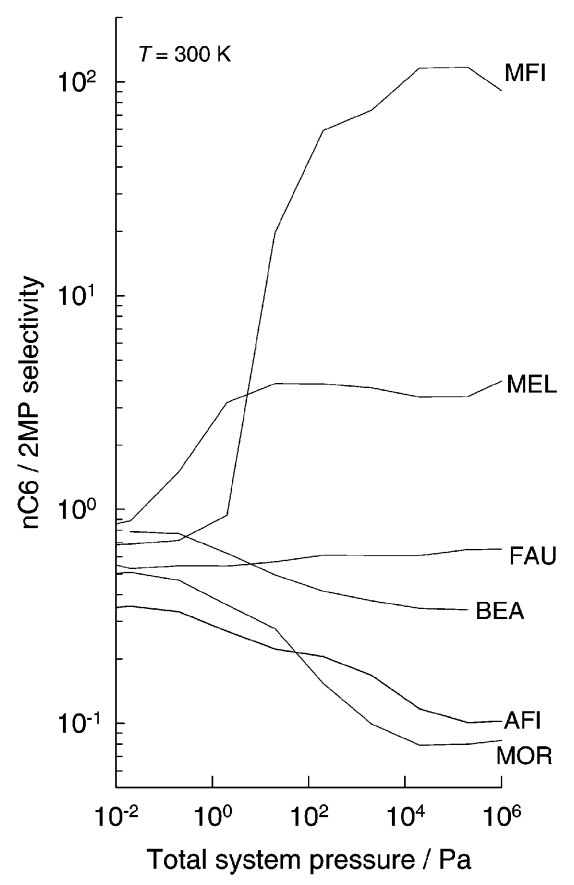

Fig. 16 CBMC simulations of sorption selectivity in a 50:50 mixture of nC6-2MP at $300 \mathrm{~K}$ in various zeolite structures. 
(a) CBMC simulations of 5-component loadings in AFI

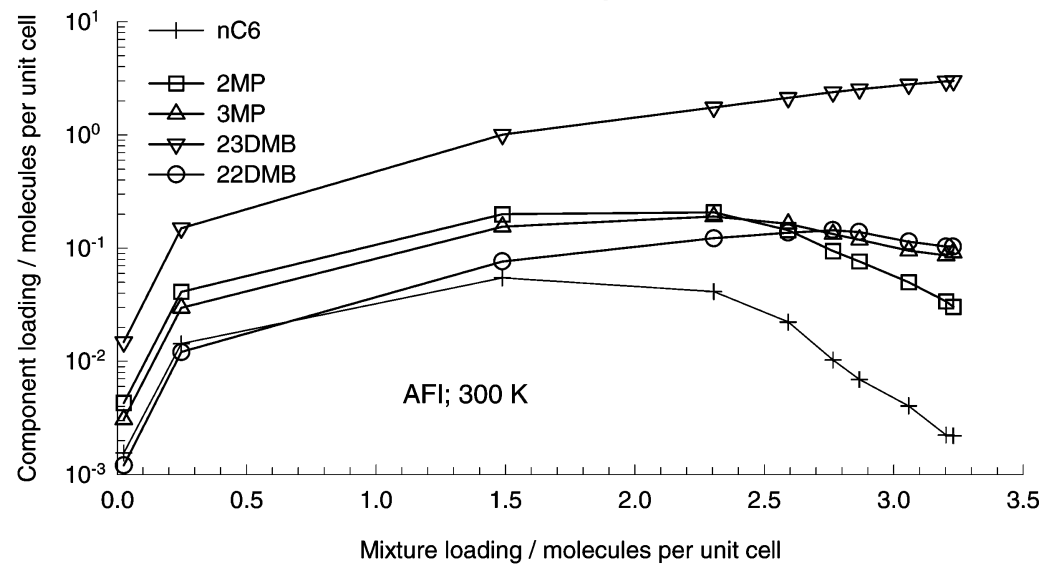

(b) CBMC simulations of 5-component loadings in MOR

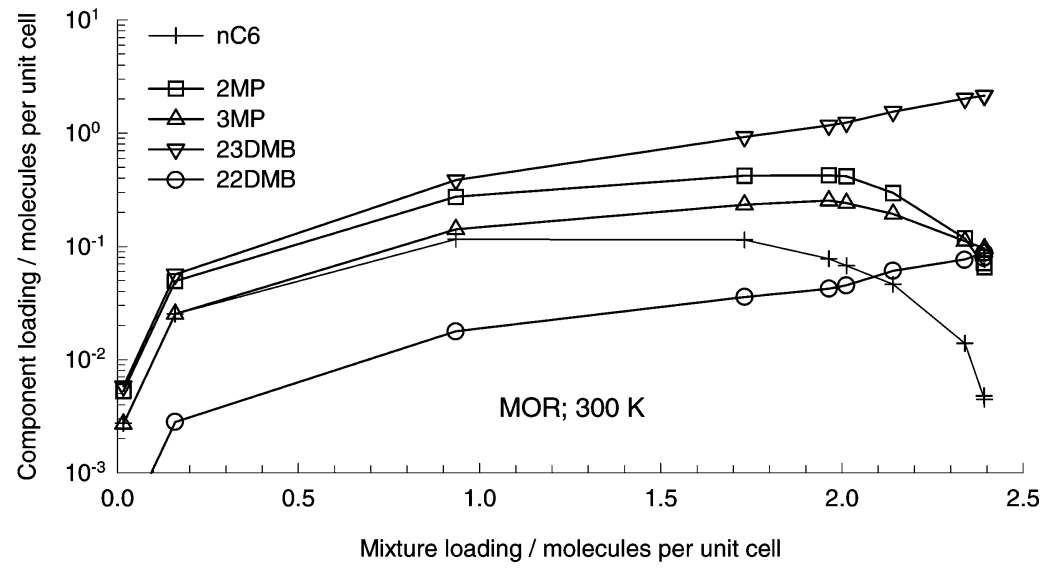

Fig. 17 CBMC simulations of component loadings for equimolar mixture of $\mathrm{nC}_{6}, 2 \mathrm{MP}, 3 \mathrm{MP}, 22 \mathrm{DMB}$ and $23 \mathrm{DMB}$ at $300 \mathrm{~K}$ in (a) $\mathrm{AFI}$ and (b) MOR.

ities; see Figs. 10 and 11. The molecule of the smaller size is preferentially adsorbed at high loadings because the few empty voids can be more easily filled up. For sorption of linear and mono-branched isomers in MFI the selectivity increases in favour of the linear isomer for mixture loadings $>4$; see Figs. $12-15$. This is due to configurational entropy effects. This effect is so strong that the branched alkanes are virtually excluded from the MFI matrix at saturation loadings. The configurational entropy concept can be exploited in practice to separate a mixture of linear and branched isomers in the 5-7 $\mathrm{C}$ atom range. ${ }^{28}$

In AFI and MOR zeolites, consisting of cylindrical channels, the length entropy effect comes into play and this favours the sorption of the isomer with the lower molecular length; see Figs 16 and 17.

For any separation task, CBMC simulations can be used to screen promising zeolite structures on the basis of their sorption selectivities at equilibrium; see e.g. Fig. 16. The more conventionally used method of using Henry coefficients to screen zeolites can lead to pessimistic conclusions because it does not reflect the subtle entropy effects that manifest at higher loadings.

There are many separations using zeolites that rely on differences in the rates of diffusion, rather than on differences in sorption loadings at equilibrium. Even in such cases, sorption isotherms are required because sorption and diffusion phenomena are intertwined, as has been shown by Krishna and Paschek. $^{3}$

Finally, we would like to add a note of caution with regard to the predictions of the sorption isotherms from CBMC simulations. These simulations pertain to ideal, rigid, crystalline zeolite structures, free from defects. In practice, zeolites are almost never defect free and there is some flexibility in the lattice; these may cause significant deviations between simulations and experiment. ${ }^{41}$ For zeolite process design, therefore, experiments are indispensible; the role of molecular simulations in this case would be to steer the experimental effort in fruitful directions.

\section{Acknowledgement}

The authors gratefully acknowledge grants from the Netherlands Organization for Scientific Research (NWO-CW).

\section{References}

1 D. M. Ruthven, Principles of Adsorption and Adsorption Processes, John Wiley, New York, 1984.

2 J. Kärger and D. M. Ruthven, Diffusion in zeolites and other microporous solids, Wiley, New York, 1992.

3 R. Krishna and D. Paschek, Sep. Purif. Technol., 2000, 21, 111.

4 K. Huddersman and M. Klimczyk, A.I.Ch.E. J., 1996, 42, 405.

5 R. S. Haizmann, F. M. Hibbs and S. Raghuram, US 5043525 to UOP, Inc., 1993.

6 H. W. Dandekar, G. A. Funk and H. A. Zinnen, US 6069289 to UOP, Inc., 2000.

7 M. S. Sun, O. Talu and D. B. Shah, J. Phys. Chem., 1996, 100, 17276.

8 L. V. C. Rees and D. Shen, Adsorption of gases in zeolite molecular sieves,ch. 13, pp. 579-631 in Introduction to Zeolite Science and Technology; ed. H. van Bekkum, E. M. Flanigen, P. A. Jacobs and J. C. Jansen; Studies in Surface Science and Catalysis, No. 137 2001, Elsevier, Amsterdam.

9 C. L. Cavalcante Jr. and D. M. Ruthven, Ind. Eng. Chem. Res., 1995, 35, 177. 
10 L. Boulicaut, S. Brandani and D. M. Ruthven, Microporous Mesoporous Mat., 1998, 25, 81.

11 W. Zhu, F. Kapteijn, B. van der Linden and J. A. Moulijn, Phys. Chem. Chem. Phys., 2001, 3, 1755.

12 O. Talu, Adv. Colloid Interf., 1998, 76-77, 227.

13 A. H. Fuchs and A. K. Cheetham, J. Phys. Chem. B, 2001, 105, 7375.

14 B. Smit and R. Krishna, Curr. Opin. Solid State Mat., 2001, 5, 455.

15 D. Frenkel and B. Smit, Understanding molecular simulations: from algorithms to applications, 2nd edn, Academic Press, San Diego, 2002.

16 T. J. H. Vlugt, M. G. Martin, J. I. Siepmann, B. Smit and R. Krishna, Mol. Phys., 1998, 94, 727.

17 T. J. H. Vlugt, R. Krishna and B. Smit, J. Phys. Chem. B, 1999, 103, 1102 .

18 J. I. Siepmann, M. G. Martin, C. J. Mundy and M. L. Klein, Mol. Phys., 1997, 90, 687.

19 M. D. Macedonia and E. J. Maginn, Fluid Phase Equilib., 1999, 158-160, 19.

20 A. G. Bezus, A. V. Kiselev, A. A. Lopatkin and P. Q. Du, J.Chem.Soc., Faraday Trans. 2, 1978, 74, 367.

21 T. J. Grey, K. P. Travis, J. D. Gale and D. Nicholson, Microporous Mesoporous Mat., 2001, 48, 203.

22 Z. Du, G. Manos, T. J. H. Vlugt and B. Smit, A.I.Ch.E J., 1998, 44, 1756.

23 T. J. H. Vlugt, W. Zhu, F. Kapteijn, J. A. Moulijn B. Smit and R. Krishna, J. Am. Chem. Soc., 1998, 120, 5599.

24 R. Krishna and D. Paschek, Phys. Chem. Chem. Phys., 2001, 3, 453.

25 M. S. Sun, D. B. Shah, H. H. Xu and O. Talu, J. Phys. Chem. B, 1998, 102, 1466
26 W. Zhu, F. Kapteijn and J. A. Moulijn, Phys. Chem. Chem. Phys., 2000, 2, 1989.

27 M. Schenk, S. L. Vidal, T. J. H. Vlugt, B. Smit and R. Krishna, Langmuir, 2001, 17, 1558.

28 S. Calero, B. Smit and R. Krishna, Phys. Chem. Chem. Phys., 2001, 3, 4390.

29 R. Krishna, B. Smit and T. J. H. Vlugt, J.Phys.Chem. A, 1998, 102, 7727.

30 R. Krishna and B. Smit, Chem. Innov., 2001, 31(1), 27.

31 B. Smit and T. L. M. Maesen, Nature, 1995, 374, 42.

32 J. Talbot, A.I.Ch.E. J., 1997, 43, 2471.

33 J. F. Denayer, G. V. Baron, J. A. Martens and P. A. Jacobs, J.Phys.Chem. B, 1998, 102, 3077.

34 K. Huddersman and M. Klimczyk, AIChE J., 1996, 42, 405.

35 J. Van de Graaf, F. Kapteijn and J. A. Moulijn, A.I.Ch.E. J., 1999, 45, 497.

36 F. Kapteijn, J. A. Moulijn and R. Krishna, Chem. Eng. Sci., 2000, 55, 2923.

37 W. J. W. Bakker, Structured systems in gas separation, $\mathrm{PhD}$ dissertation, Delft University of Technology, Delft, 1999.

38 D. S. Santilli, J. Catal., 1986, 99, 335.

39 S. Calero, B. Smit and R. Krishna, J. Catal., 2001, 202, 395.

40 R. W. Carr and H. W. Dandekar, Adsorption with reaction, inReactive Separation Processes, ed. S. Kulprathipanja, Taylor \& Francis, Philadelphia 2002.

41 L. A. Clark and R. Q. Snurr, Chem. Phys. Lett., 1999, 308, 155. 